\title{
6-Oxyindan-1-ones with amino acid fragments
}

\author{
Serhiy V. Shilin*, Illya N. Lenko, Olga V. Shablykina, Volodymyr P. Khilya \\ Department of Chemistry, Taras Shevchenko National University of Kyiv, \\ Volodymyrska Street, 64/13, Kyiv 01601, Ukraine \\ varang77@ukr.net
}

Keywords: 6-hydroxyindan-1-one, activated ester method, N-hydroxysuccinimide, amino acid derivatives.

New indan-1-one derivatives ( 8 examples) with amino acid fragments were synthesized through the $\mathrm{N}$-acylation of the amino acids by 2-(3-oxo-2,3-dihydro- $1 H$-inden-5-yloxy)acetic acid using the method of activated $N$-hydroxysuccinimide esters. To obtain corresponding methyl esters ( 2 examples) two ways were possible: the $N$-acylation of the amino acid methyl esters by 2-(3-oxo2,3-dihydro-1-inden-5-yloxy)acetic acid through the activated imidazole derivatives or methylation of the carboxylic function of preformed $N-\{[(1-$ oxoindan-6-yl)oxy $]$ acetyl $\}$ amino acids.

\section{Introduction}

Compounds containing an indane fragment are objectively widely represented in nature. The variability of such structures is provided by different types of substituents, not only in the benzene ring, but also those presented in positions 1-3: this fragment of the molecule can be saturated or can hold a double bond, or functional groups mainly hydroxy or keto groups.

Among natural indanes, probably, the class of pterosins is most meticulously studied (Figure 1). These sesquiterpenes, contained in various fern species, have interested researchers for their bioactivity $[1,2]$, but, unfortunately, many of the pterosins are highly toxic. So, it is not surprising that in recent years there have been a lot of experiments to creation bioactive substances with an indane fragment devoid of this shortcoming, and many tries had been successful [3-5].

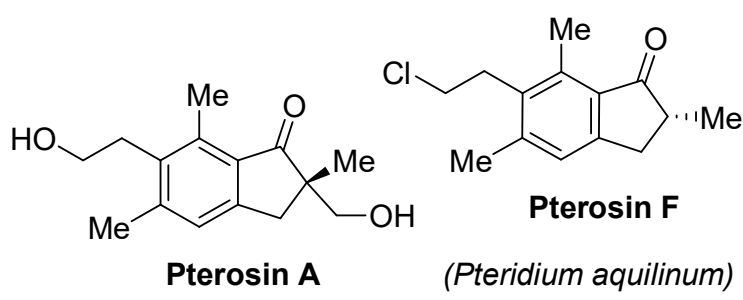

Figure 1. Pterosins - naturally occurring indan-1-ones.

In particular, it has been interesting and beneficial for medicinal chemistry to combine the indane cycle with the amino acid residue in structures of varying complexity. Among these molecules (Figure 2) we can find antihypertensive agent 1 [3], compound 2 with antidiabetic activity [4], and antipsoriasis agent $\mathbf{3}$ [5]. That is why the purpose of the present work 
was to obtain new amino acid derivatives of indane.

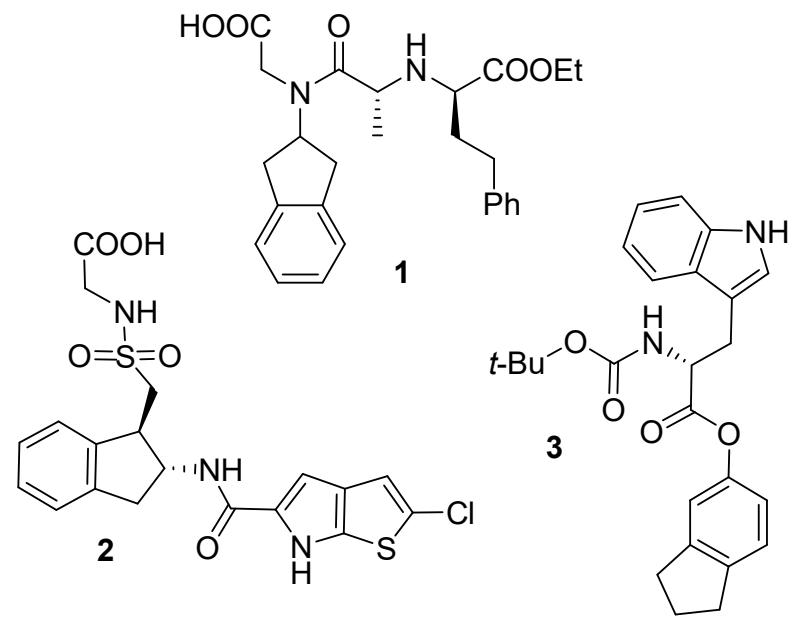

Figure 2. Bioactive indane derivatives with amino acid fragments.

As a base object for modification we chose 6-hydroxyindan-1-one 4. Firstly, its molecule contains a phenolic $\mathrm{OH}$ group, convenient for connection of the amino acid residue via an amide bond with a hydroxylacetic linker; furthermore, exactly the indan-1-one system is observed in natural high-bioactive pterosins (Figure 1). In addition, indanones are a convenient material for conversion into a variety of related derivatives: indanes, hydroxyand aminoindanes.

Studies of 6-hydroxyindan-1-on began in the 1920s with the works of Ingold and Piggott [6], and while in subsequent years the number of publications devoted to derivatives of the indane series has increased significantly [7]; and in some studies the synthetic possibilities of this molecule was been shown. For example, the using of 6-hydroxyindan-1-one 4 proved to be convenient for the preparation of Ladostigil (TV-3326) (Scheme 1), such substance demonstrates excellent results in the treatment of neurodegenerative disorders (Parkinsonism, Alzheimer's disease) [8].<smiles>O=C1CCc2ccc(O)cc21</smiles>

4

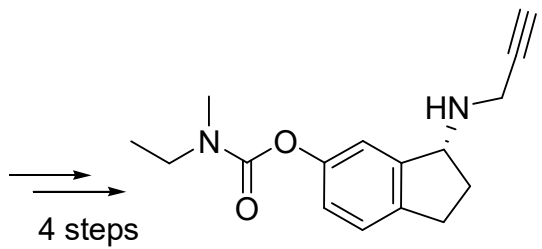

Ladostigil
Scheme 1. 6-Hydroxyindan-1-one in Ladostigil synthesis

Known at first only as a synthetic compound, indanone 4 was recently isolated in nature: beside the pyridine alkaloids, polyphenols and cinnamic acids, this compound was isolated from the plant Scrophularia ningpoensis, known in traditional Chinese medicine due to myocardioprotective properties [9].

\section{Results and discussion}

Modification of indanone 4 with a hydroxyacetic fragment was carried out according to a scheme analogous to that described in the early publication [10] (Scheme 2); however for hydrolysis of ester 5 not acid but basic medium was used. (It had been noted that water solution of potash was implemented for such reaction; and using of aqueous alkali isn't recommended because the indanone system tends to condense in an alkaline medium [11]). 


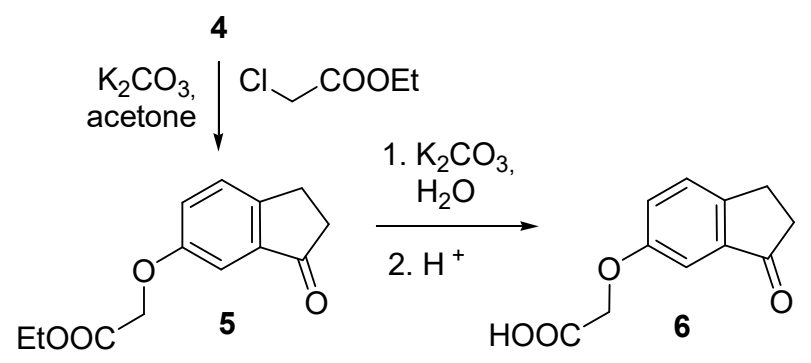

Scheme 2. The synthesis of 2-(3-oxo-2,3-dihydro$1 H$-inden-5-yloxy)acetic acid

To create the amide bond of acid 6 with the $\mathrm{NH}_{2}$ group of amino acids, the classical method of activated $N$-hydroxysuccinimide esters was used [12, 13] (Scheme 3). This method makes it possible to obtain acylating agents (succinimide esters 7) and to acylate the $\mathrm{NH}_{2}$ group of the amino acid thought consecutive addition of reagents as "one-pot" synthesis; and the process conditions are mild, which is very important in case of acid 6 modification.

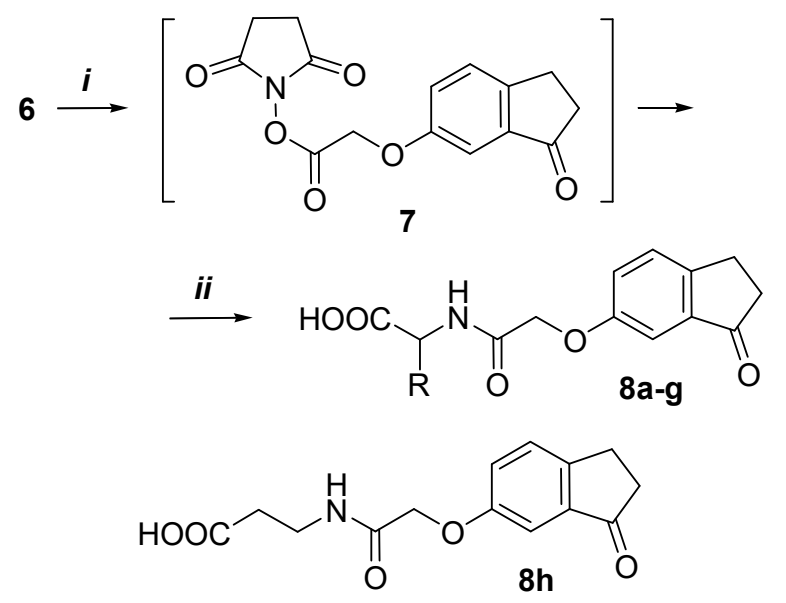

i: 1. DCC, abs. dioxane, rt; 2. N-hydroxysuccinimide ii: 1. amino acid, $\mathrm{NaHCO}_{3}$, dioxane : water $1: 1, \mathrm{rt} ; 2 . \mathrm{HCl}$

Scheme 3. The $N$-acylation of the amino acids by 2-(3-oxo-2,3-dihydro-1H-inden-5-yloxy)acetic acid thought the method of $N$-hydroxysuccinimide esters ( $\mathrm{R}$ see Table 1).
The benefits of $N$-hydroxysuccinimide esters using became more apparent by a comparison it with another method we tried. Namely, some amino acid derivatives of indanone were obtained in reaction of methyl esters of amino acids and the activated imidazole derivative of acid 6 [13] (Scheme 4).

The reactions wich were presented on Scheme 4 also carried out in a "one-pot" variation; and the yields of methyl esters 10a,c can be considered suitable, but they are lower to the yields of products $\mathbf{8 a}, \mathbf{c}$.

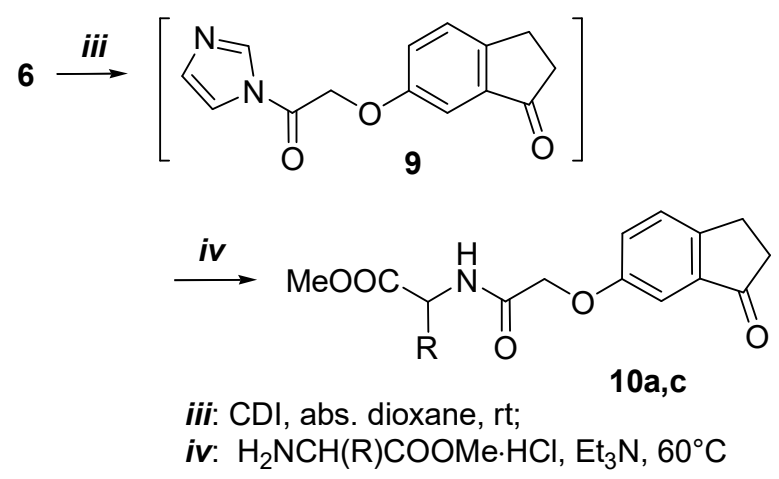

Scheme 4. The $N$-acylation of the amino acids methyl esters by 2-(3-oxo-2,3-dihydro-1H-inden-5-yloxy)acetic acid thought the activated imidazole derivatives ( $R$ see Table 1).

An alternative tactic to the obtaining of compounds 10a,c could be implemented for the next way: the preparation of the amino acid derivative 8 through the stage of $N$-hydroxysuccinimide ester, and following methylation of the carboxylic function with methyl iodide. To compare the efficiency of this procedure with the reactions on Scheme 4, the methionine derivative 10c (Scheme 4) was also obtained by 
methylation of the corresponding derivative 8c (Scheme 5).

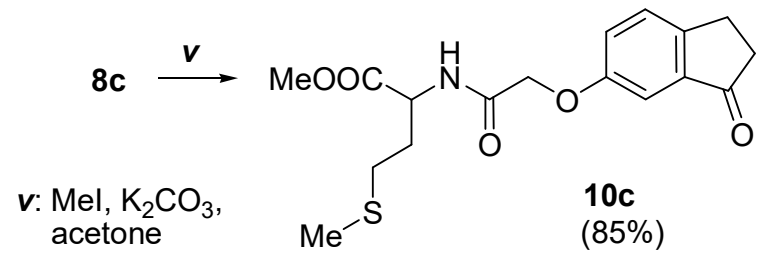

Scheme 5. Synthesis of ester 10c by methylation of acid 8c.

Table 1. List, yields and melting points of the products $\mathbf{8 , 1 0}$

\begin{tabular}{|c|c|c|c|}
\hline $\begin{array}{l}\text { Pro- } \\
\text { duct }\end{array}$ & $\begin{array}{c}\text { Initial amino acid } \\
(\mathrm{R})\end{array}$ & m.p., ${ }^{\circ} \mathbf{C}$ & $\begin{array}{c}\text { Yeild, } \\
\%\end{array}$ \\
\hline $\mathbf{8 a}$ & Gly $(\mathrm{H})$ & 196-197 & 57 \\
\hline $8 b$ & $\begin{array}{c}N l e \\
(n-\mathrm{Bu})\end{array}$ & $133-134$ & 28 \\
\hline $8 c$ & $\begin{array}{c}M e t \\
\left(\mathrm{CH}_{2} \mathrm{CH}_{2} \mathrm{SCH}_{3}\right)\end{array}$ & $71-72$ & 80 \\
\hline $8 d$ & Phe (Bn) & $142-143$ & 56 \\
\hline $8 e$ & $\begin{array}{c}T y r \\
\left(\mathrm{CH}_{2}\left(4-\mathrm{OHC}_{6} \mathrm{H}_{4}\right)\right)\end{array}$ & 197-198 & 26 \\
\hline $8 f$ & $\begin{array}{c}\operatorname{Trp} \\
\left(\mathrm{CH}_{2}(\text { indol-3-yl })\right)\end{array}$ & $148-149$ & 57 \\
\hline $8 g$ & $\begin{array}{c}C y t \\
\left(\left(\mathrm{CH}_{2}\right)_{3} \mathrm{NHC} \mathrm{NH}\right) \\
\left.\mathrm{NH}_{2}\right)\end{array}$ & $185 \mathrm{dec}$ & 61 \\
\hline $8 \mathrm{~h}$ & $\beta$-Ala & 195-196 & 48 \\
\hline $10 \mathrm{a}$ & $G l y(\mathrm{H})$ & $64-65$ & 43 \\
\hline $10 \mathrm{c}$ & $\begin{array}{c}M e t \\
\left(\mathrm{CH}_{2} \mathrm{CH}_{2} \mathrm{SCH}_{3}\right)\end{array}$ & $89-90$ & $\begin{array}{l}52^{*} / \\
85^{* *}\end{array}$ \\
\hline
\end{tabular}

In this case, the total yield of the substance 10c from the initial acid 6 after two stages was $68 \%$, which is higher than the yield following the Scheme 4 using (52\%). Therefore a strategy based on the production of amino acid derivatives of indanone type 8 should be considered most suitable not only because these products can be obtained easily and in high yields, but also because compounds 8 can be very convenient raw materials for the synthesis of the corresponding methyl esters $\mathbf{1 0}$.

\section{Experimental part}

Reaction flow and identity of obtained compounds was controlled with TLC on Merck $\mathrm{F}_{254}$ plates using chloroform : methanol (19:1, $v / v$ ) system as eluents.

${ }^{1} \mathrm{H},{ }^{13} \mathrm{C}$ NMR spectra were recorded at Varian 400 spectrometer operating at $400 \mathrm{MHz}$ frequency for ${ }^{1} \mathrm{H}$ and $100 \mathrm{MHz}$ for ${ }^{13} \mathrm{C}$ experiments. NMR chemical shifts are reported in ppm, in the $\delta$ scale and are referenced using TMS as internal standard.

IR spectra were recorded on a Perkin Elmer BX II spectrometer in $\mathrm{KBr}$ pellets.

Melting points were determined using a Kofler-type Leica Galen III micro hot stage microscope and uncorrected.

General procedure for $N$-acylation of the amino acid by acid 6 through the method of $\boldsymbol{N}$-hydroxysuccinimide esters. To a solution of $0.21 \mathrm{~g}(1 \mathrm{mmol})$ of acid 6 and $0.13 \mathrm{~g}$ (1.1 mmol) of $N$-hydroxysuccinimide in $10 \mathrm{ml}$ of absolute dioxane at room temperature and with vigorous stirring $0.23 \mathrm{~g}(1.1 \mathrm{mmol})$ of dicyclohexylcarbodiimide was added. The reaction mixture was stirred at room 
temperature for $2-3 \mathrm{~h}$ until an activated ester was formed (TLC monitoring); then a solution of $1.1 \mathrm{mmol}$ of the corresponding amino acid and $0.14 \mathrm{~g}(1.67 \mathrm{mmol})$ of $\mathrm{NaHCO}_{3}$ in $10 \mathrm{ml}$ of water was added. The reaction mixture was stirred at room temperature for $2-3 \mathrm{~h}$ (TLC monitoring); and after the process was finished the dicyclohexylurea precipitate was filtered off. The filtrate was poured into $50 \mathrm{ml}$ of water and the solution was acidified $(\mathrm{pH} \mathrm{4-5)}$ with dilute $\mathrm{HCl}$. The precipitate formed was filtered off, recrystallized from mixture $i-\mathrm{PrOH}$ - water 1:1.

$N$ - $\{[(1-$ oxoindan-6-yl)oxy $]$ acetyl $\}$ glycine 8a. ${ }^{1} \mathrm{H}$ NMR (DMSO-d 6 ): 2.62 (br. s, 2H, $\mathrm{H}_{2}-3$ ), 3.00 (br. s, 2H, $\mathrm{H}_{2}-2$ ), 3.82 (br. s, $2 \mathrm{H}$, $\mathrm{NHCH}_{2}$ ), 4.59 (s, $2 \mathrm{H}, \mathrm{OCH}_{2} \mathrm{CO}$ ), 7.10 (br. s, 1H, H-7), 7.33 (br. d, $J=8.0 \mathrm{~Hz}, 1 \mathrm{H}, \mathrm{H}-5$ ), 7.49 (d, $J=8.0 \mathrm{~Hz}, 1 \mathrm{H}, \mathrm{H}-4), 8.48$ (br. s, 1H, NH). ${ }^{13} \mathrm{C}$ NMR (DMSO-d d $_{\text {): }} 25.3,37.2,41.1,67.6$, 107.0, 124.2, 128.5, 138.4, 149.1, 157.8, 168.6, 171.7, 206.8. IR ( $\left.\mathrm{KBr}, v, \mathrm{~cm}^{-1}\right): 3529,3445$, $3288,1729,1664,1552,1494,1311,1222$, $1071,836$.

$N-\{[(1-o x o i n d a n-6-y l) o x y]$ acetyl $\}$ nor-

leucine 8b. ${ }^{1} \mathrm{H}$ NMR (DMSO- $\mathrm{d}_{6}: \mathrm{CCl}_{4} \quad$ 1:1): $0.89\left(\mathrm{~m}, 3 \mathrm{H}, \mathrm{NHCH}\left[\left(\mathrm{CH}_{2}\right)_{3} \mathrm{CH}_{3}\right]\right), 1.28(\mathrm{~m}, 4 \mathrm{H}$, $\left.\mathrm{NHCH}\left[\mathrm{CH}_{2}\left(\underline{\mathrm{CH}_{2}}\right)_{2} \mathrm{CH}_{3}\right]\right), \quad 1.67 \quad(\mathrm{~m}, \quad 1 \mathrm{H}$, $\left.\left[\underline{\mathrm{CH}}_{\alpha}\left(\mathrm{CH}_{2}\right)_{2} \mathrm{CH}_{3}\right]\right), 1.77\left(\mathrm{~m}, 1 \mathrm{H}, \mathrm{NHCH}\left[\underline{\mathrm{CH}}_{\beta}-\right.\right.$ $\left.\left.\left(\mathrm{CH}_{2}\right)_{2} \mathrm{CH}_{3}\right]\right), 2.64$ (br. t, $J=4.3 \mathrm{~Hz}, 2 \mathrm{H}, \mathrm{H}_{2}-3$ ), 3.07 (br. t, $J=4.3 \mathrm{~Hz}, 2 \mathrm{H}, \mathrm{H}_{2}-2$ ) 4.27 (m, $1 \mathrm{H}$, $\mathrm{NH} \underline{\mathrm{CH}}[n-\mathrm{Bu}]), \quad 4.54 \quad(\mathrm{~d}, \quad J=14.6 \mathrm{~Hz}, \quad 1 \mathrm{H}$, $\left.\mathrm{OCH}_{\alpha} \mathrm{CO}\right), 4.59$ (d, $J=14.6 \mathrm{~Hz}, 1 \mathrm{H}, \mathrm{OCH}_{\beta} \mathrm{CO}$ ), $7.11 \quad(\mathrm{~d}, \quad J=2.2 \mathrm{~Hz}, \quad 1 \mathrm{H}, \quad \mathrm{H}-7), \quad 7.30 \quad$ (dd, $\left.{ }^{3} J=8.0 \mathrm{~Hz},{ }^{4} J=2.2 \mathrm{~Hz}, \mathrm{H}-5\right), 7.46$ (d, $J=8.0 \mathrm{~Hz}$, $\mathrm{H}-4), 8.09(\mathrm{~m}, 1 \mathrm{H}, \mathrm{NH}), 12.57$ (br. s, 1H, $\mathrm{COOH}) . \quad{ }^{1} \mathrm{H} \mathrm{NMR} \quad\left(\mathrm{CDCl}_{3}\right): 0.90 \quad(\mathrm{~m}, \quad 3 \mathrm{H}$, $\left.\mathrm{NHCH}\left[\left(\mathrm{CH}_{2}\right)_{3} \mathrm{CH}_{3}\right]\right), \quad 1.34 \quad(\mathrm{~m}, \quad 4 \mathrm{H}$, $\left.\mathrm{NHCH}\left[\mathrm{CH}_{2}\left(\underline{\mathrm{CH}}_{2}\right)_{2} \mathrm{CH}_{3}\right]\right), \quad 1.80 \quad(\mathrm{~m}, \quad 1 \mathrm{H}$, $\left.\left[\underline{\mathrm{CH}}_{\alpha}\left(\mathrm{CH}_{2}\right)_{2} \mathrm{CH}_{3}\right]\right), 1.97\left(\mathrm{~m}, 1 \mathrm{H}, \mathrm{NHCH}\left[\underline{\mathrm{CH}}_{\beta}-\right.\right.$ $\left.\left.\left(\mathrm{CH}_{2}\right)_{2} \mathrm{CH}_{3}\right]\right), 2.75$ (br. t, $J=5.0 \mathrm{~Hz}, 2 \mathrm{H}, \mathrm{H}_{2}-3$ ), 3.11 (br. t, $\left.J=5.0 \mathrm{~Hz}, 2 \mathrm{H}, \mathrm{H}_{2}-2\right), 4.58(\mathrm{~s}, 2 \mathrm{H}$, $\left.\mathrm{OCH}_{2} \mathrm{CO}\right), 4.72(\mathrm{~m}, 1 \mathrm{H}, \mathrm{NH} \underline{\mathrm{CH}}[n-\mathrm{Bu}]), 7.00$ (br. d, $J=7.2 \mathrm{~Hz}, 1 \mathrm{H}, \mathrm{NH}$ ), 7.28 (m, 2H, H-5,7), $7.45(\mathrm{~d}, J=8.4 \mathrm{~Hz}, 1 \mathrm{H}, \mathrm{H}-4) .{ }^{13} \mathrm{C} \mathrm{NMR}\left(\mathrm{CDCl}_{3}\right)$ : $13.8,22.3,25.2,27.3,37.0,51.7,56.6,67.5$, 107.3, 123.6, 127.9, 138.4, 149.2, 156.8, 168.0, 171.3, 206.7. IR ( $\left.\mathrm{KBr}, v, \mathrm{~cm}^{-1}\right)$ : 3380,2958 , 1700, 1540, 1274, 1220, 1187, 1062, 836.

\section{$N$-\{[(1-oxoindan-6-yl)oxy]acetyl $\}$ -}

methionine 8c. ${ }^{1} \mathrm{H}$ NMR (DMSO- $\mathrm{d}_{6}: \mathrm{CCl}_{4}$ 1:1): 1.91-2.08 (m, 5H, NHCH[CH$\left.\left.{ }_{2} \underline{\mathrm{CH}}_{2} \underline{\mathrm{SCH}}_{3}\right]\right), 2.43$ $\left(\mathrm{m}, 2 \mathrm{H}, \mathrm{NHCH}\left[\underline{\mathrm{CH}}_{2} \mathrm{CH}_{2} \mathrm{SCH}_{3}\right]\right), 2.64(\mathrm{~m}, 2 \mathrm{H}$, $\left.\mathrm{H}_{2}-3\right), 3.07\left(\mathrm{~m}, 2 \mathrm{H}, \mathrm{H}_{2}-2\right), 4.42(\mathrm{~m}, 1 \mathrm{H}$, $\left.\mathrm{NHCH}\left[\left(\mathrm{CH}_{2}\right)_{2} \mathrm{SCH}_{3}\right]\right), 4.55\left(\mathrm{~m}, 2 \mathrm{H}, \mathrm{OCH}_{2} \mathrm{CO}\right)$, 7.12 (br. s, $1 \mathrm{H}, \mathrm{H}-7$ ), 7.29 (br. d, $J=8.2 \mathrm{~Hz}$, H-5), 7.44 (d, J=8.2 Hz, H-4), 8.17 (br. d, $J=7.0 \mathrm{~Hz}, \quad 1 \mathrm{H}, \quad \mathrm{NH}) .{ }^{13} \mathrm{C}$ NMR $\left(\right.$ DMSO-d $_{6}$ ): 15.2, 25.4, 30.4, 31.0, 37.3, 51.3, 67.5, 106.8, $124.3,128.4,138.4,149.0,158.0,168.4,173.7$, 206.5. IR (KBr, $\left.v, \mathrm{~cm}^{-1}\right): 3501,3400,2935$, 1670, 1519, 1446, 1309, 1256, 1208, 1063, 839.

\section{$N$-\{[(1-oxoindan-6-yl)oxy $]$ acetyl $\}$ -}

phenylalanine 8d. ${ }^{1} \mathrm{H}$ NMR ( $\left.\mathrm{CDCl}_{3}\right): 2.75$ (br. t, $J=5.2 \mathrm{~Hz}, 2 \mathrm{H}, \mathrm{H}_{2}-3$ ), 3.11 (br. t, $J=5.2 \mathrm{~Hz}, 2 \mathrm{H}$, $\left.\mathrm{H}_{2}-2\right), 3.20\left(\mathrm{dd},{ }^{2} J=14.2 \mathrm{~Hz},{ }^{3} J=6.4 \mathrm{~Hz}, 1 \mathrm{H}\right.$, $\left.\mathrm{NHCH}\left[\underline{\mathrm{CH}}_{\alpha} \mathrm{Ph}\right]\right), \quad 3.26 \quad\left(\mathrm{dd}, \quad{ }^{2} J=14.2 \mathrm{~Hz}\right.$, 
$\left.{ }^{3} J=6.4 \mathrm{~Hz}, \quad 1 \mathrm{H}, \quad \mathrm{NHCH}\left[\underline{\mathrm{CH}}_{\beta} \mathrm{Ph}\right]\right), \quad 4.50 \quad(\mathrm{~d}, \quad 149.0,156.5,157.9,168.1,173.4,201.7$. IR $\left.J=15.0 \mathrm{~Hz}, 1 \mathrm{H}, \mathrm{OCH}_{\alpha} \mathrm{CO}\right), 4.55\left(\mathrm{~d}, J=15.0 \mathrm{~Hz}, \quad\left(\mathrm{KBr}, \mathrm{v}, \mathrm{cm}^{-1}\right): 3384,2918,1720,1703,1630\right.$, $\left.1 \mathrm{H}, \quad \mathrm{OCH}_{\beta} \mathrm{CO}\right), 4.98 \quad(\mathrm{q}, \quad J=6.4 \mathrm{~Hz}, \quad 1 \mathrm{H}, \quad 1544,1491,1438,1278,1205,1063,914,836$, $\mathrm{NH} \underline{\mathrm{CH}}[\mathrm{Bn}]), 6.95$ (br. d, J=8.2 Hz, 1H, NH), 794.

7.13-7.19 (m, 5H, Ph), $7.28(\mathrm{~m}, 2 \mathrm{H}, \mathrm{H}-5,7)$, $N$ - $\{[(1-$ oxoindan-6-yl)oxy $]$ acetyl $\}-$

$7.42(\mathrm{~d}, \quad J=8.0 \mathrm{~Hz}, \quad 1 \mathrm{H}, \quad \mathrm{H}-4) . \quad{ }^{1} \mathrm{H}$ NMR tryptophan 8f. ${ }^{1} \mathrm{H}$ NMR (DMSO-d 6 ): 2.64 (br. t,

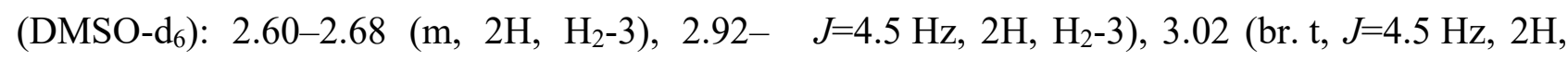
$3.05\left(\mathrm{~m}, 3 \mathrm{H}, \mathrm{H}_{2}-2, \mathrm{NHCH}\left[\underline{\mathrm{CH}}_{\alpha} \mathrm{Ph}\right]\right), 3.26$ (dd, $\left.\mathrm{H}_{2}-2\right), 3.12-3.31$ (m, 2H, NHCH$\left[\underline{\mathrm{CH}}_{2}\right.$ (indol-3$\left.\left.\left.\left.{ }^{2} J=13.2 \mathrm{~Hz},{ }^{3} J=3.6 \mathrm{~Hz}, 1 \mathrm{H}, \quad \mathrm{NHCH}\left[\underline{\mathrm{CH}}_{\beta} \mathrm{Ph}\right]\right), \quad \mathrm{ll}\right)\right]\right), \quad 4.49-4.65 \quad\left(\mathrm{~m}, \quad 3 \mathrm{H}, \quad \mathrm{OCH}_{2} \mathrm{CO}\right.$, 4.45-4.61 (m, 3H, $\mathrm{OCH}_{2} \mathrm{CO}, \mathrm{NH} \underline{\mathrm{CH}}[\mathrm{Bn}], 7.05 \quad \mathrm{NHCH}\left[\mathrm{CH}_{2}\right.$ (indol-3-yl)]), 6.97 (br. t, $J=7.8 \mathrm{~Hz}$, (br. s, 1H, H-7), 7.11-7.29 (m, 6H, H-5, Ph), 1H, H-5' (indol-3-yl)), 7.00-7.11 (m, 2H, 7.47 (d, J=8.2 Hz, 1H, H-4), 8.31 (br. s, 1H, H-2',6'), 7.14 (br. s, 1H, H-7), 7.22 (br. d, NH), 12.87 (br. s, 1H, COOH). ${ }^{13} \mathrm{C} \mathrm{NMR} \mathrm{J=7.8} \mathrm{Hz,} \mathrm{H-5),} 7.36$ (d, J=7.8 Hz, 1H, H-4' (DMSO-d 6 ): 25.4, 37.0, 37.2, 53.9, 67.4, 106.9, $124.1,127.1,128.5,128.9,129.7,138.1 \times 2$, $138.4 \times 2,149.0,157.9,168.2,173.3,206.6$. IR $\left(\mathrm{KBr}, \mathrm{v}, \mathrm{cm}^{-1}\right): 3412,3266,2930,1673 \mathrm{br}$, 1544, 1490, 1306, 1278, 1222, 1186, 1069, 845, 693.

$N$-\{[(1-oxoindan-6-yl)oxy $]$ acetyl $\}$ (indol-3-yl)), 7.46 (d, J=7.8 Hz, H-4), 7.56 (d, $J=7.8 \mathrm{~Hz}, 1 \mathrm{H}, \mathrm{H}-7^{\prime}$ (indol-3-yl)), $7.96(\mathrm{~m}, 1 \mathrm{H}$, $\mathrm{NH}), 10.79$ (s, 1H, NH (indol-3-yl)). ${ }^{13} \mathrm{C} \mathrm{NMR}$ (DMSO-d 6 ): 25.4, 27.5, 37.3, 53.5, 67.5, 107.1, $110.3,112.1,118.8,119.1,121.6,124.0,124.4$, $127.9,128.5,136.7,138.4,149.0,157.8,168.0$, 173.8, 206.7. IR ( $\left.\mathrm{KBr}, v, \mathrm{~cm}^{-1}\right): 3406,3339$, tyrosine 8e. ${ }^{1} \mathrm{H}$ NMR (DMSO-d $\left.\mathrm{d}_{6}\right): 2.64$ (br. t, $\left.J=5.2 \mathrm{~Hz}, \quad 2 \mathrm{H}, \quad \mathrm{H}_{2}-3\right), \quad 2.86 \quad(\mathrm{~m}, \quad 1 \mathrm{H}, \quad 745$. $\left.\mathrm{NHCH}\left[\underline{\mathrm{CH}}_{\alpha}\left(4-\mathrm{HOC}_{6} \mathrm{H}_{4}\right)\right]\right), \quad 2.95 \quad(\mathrm{~m}, \quad 1 \mathrm{H}$, $\mathrm{NHCH}\left[\underline{\mathrm{CH}}_{\beta}\left(4-\mathrm{HOC}_{6} \mathrm{H}_{4}\right)\right]$ ), 3.01 (br. t, $J=5.2 \mathrm{~Hz}$, $\left.2 \mathrm{H}, \quad \mathrm{H}_{2}-2\right), \quad 4.41 \quad\left(\mathrm{~m}, \quad 1 \mathrm{H}, \quad \mathrm{NHCH}\left[\mathrm{CH}_{2}-\right.\right.$ $\left.\left.\left(4-\mathrm{HOC}_{6} \mathrm{H}_{4}\right)\right]\right), 4.54\left(\mathrm{~m}, 2 \mathrm{H}, \mathrm{OCH}_{2} \mathrm{CO}\right), 6.60$ (d, $\left.J=8.3 \mathrm{~Hz}, 2 \mathrm{H}, \mathrm{H}-3^{\prime}, 5^{\prime}\left(4-\mathrm{HOC}_{6} \mathrm{H}_{4}\right)\right), 6.96$ (d, $J=8.3 \mathrm{~Hz}, 2 \mathrm{H}, \mathrm{H}-2^{\prime}, 6^{\prime}\left(4-\mathrm{HOC}_{6} \mathrm{H}_{4}\right)$ ), 7.05 (br. s, 1H, H-7), 7.24 (br. d, J=8.2 Hz, H-5), 7.47 (d, $J=8.2 \mathrm{~Hz}, \mathrm{H}-4), 8.22$ (d, J=8.0 Hz, 1H, NH),

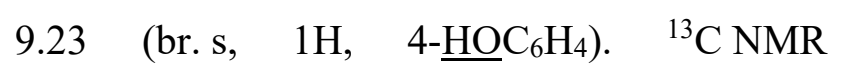
(DMSO-d 6 ): 25.4, 36.3, 37.3, 54.2, 67.5, 106.9, $115.6 \times 2,124.0,128.1,128.5,130.7 \times 2,138,4$, 1700 br, 1535, 1278, 1222, 1186, 1065, 833,

$N$ - $\{[(1-$ oxoindan-6-yl)oxy $]$ acetyl $\}$ -

citrulline 8g. ${ }^{1} \mathrm{H}$ NMR (DMSO- $\mathrm{d}_{6}: \mathrm{CCl}_{4} \quad$ 1:1): 1.37-1.46 (m, 2H, $\mathrm{NHCH}\left[\mathrm{CH}_{2} \underline{\mathrm{CH}_{2}} \mathrm{CH}_{2}-\right.$ $\left.\left.\mathrm{NHCONH}_{2}\right]\right), \quad 1.61-1.71 \quad(\mathrm{~m}, \quad 1 \mathrm{H}$, $\left.\mathrm{NHCH}\left[\underline{\mathrm{CH}}_{\alpha}\left(\mathrm{CH}_{2}\right)_{2} \mathrm{NHCONH}_{2}\right]\right), 1.75-1.85(\mathrm{~m}$, $\left.1 \mathrm{H}, \mathrm{NHCH}\left[\underline{\mathrm{CH}} \beta\left(\mathrm{CH}_{2}\right)_{2} \mathrm{NHCONH}_{2}\right]\right), 2.64$ (br. t, $\left.J=4.5 \mathrm{~Hz}, \quad 2 \mathrm{H}, \mathrm{H}_{2}-3\right), 2.95-3.01 \quad(\mathrm{~m}, 2 \mathrm{H}$, $\left.\mathrm{NHCH}\left[\left(\mathrm{CH}_{2}\right)_{2} \underline{\mathrm{CH}}_{2} \mathrm{NHCONH}_{2}\right]\right), 3.06$ (br. $\mathrm{t}$, $\left.J=4.5 \mathrm{~Hz}, \quad 2 \mathrm{H}, \mathrm{H}_{2}-2\right), 4.25-4.32 \quad(\mathrm{~m}, 1 \mathrm{H}$, $\left.\mathrm{NH} \underline{\mathrm{CH}}\left[\left(\mathrm{CH}_{2}\right)_{3} \mathrm{NHCONH}_{2}\right]\right), \quad 4.56 \quad(\mathrm{~s}, \quad 2 \mathrm{H}$, $\mathrm{OCH}_{2} \mathrm{CO}$ ), 5.93 (br. s, 1H, $\mathrm{NHCONH}_{2}$ ), 7.10 (d, 
$J=2.2 \mathrm{~Hz}, \quad 1 \mathrm{H}, \mathrm{H}-7), 7.29$ (dd, ${ }^{3} J=8.3 \mathrm{~Hz}$, formed precipitate was filtered off, $\left.{ }^{4} J=2.2 \mathrm{~Hz}, \mathrm{H}-5\right), 7.44(\mathrm{~d}, J=8.3 \mathrm{~Hz}, \mathrm{H}-4), 8.21$ recrystallized from mixture $i$-PrOH - water 1:1. $(\mathrm{d}, J=7.6 \mathrm{~Hz}, 1 \mathrm{H}, \mathrm{NH}) .{ }^{13} \mathrm{C} \mathrm{NMR}\left(\mathrm{CDCl}_{3}\right): 25.2$, Methyl ester of $N$-\{[(1-oxoindan-6-yl)$27.2,28.8,37.1,52.0,67.3,106.7,124.0,128.3$, $138.3,148.8,157.9,159.2,168.0,173.7,206.5$. IR $\left(\mathrm{KBr}, v, \mathrm{~cm}^{-1}\right): 3373,3317,2936,2880$, 1700, 1595, 1440, 1261, 1057, 845.

$N$-\{[(1-oxoindan-6-yl)oxy $]$ acetyl $\}$ $\beta$-alanine 8h. ${ }^{1} \mathrm{H}$ NMR (DMSO- $\mathrm{d}_{6}: \mathrm{CCl}_{4} \quad 1: 1$ ): 2.41 (br. t, $J=6.6 \mathrm{~Hz}, 2 \mathrm{H}, \mathrm{NHCH}_{2} \underline{\mathrm{CH}_{2}}$ ), 2.63 (br. t, $J=4.3 \mathrm{~Hz}, 2 \mathrm{H}, \mathrm{H}_{2}-3$ ), 3.05 (br. t, $J=4.3 \mathrm{~Hz}$, $\left.2 \mathrm{H}, \mathrm{H}_{2}-2\right), 3.31-3.40\left(\mathrm{~m}, 2 \mathrm{H}, \mathrm{NHCH}_{2} \mathrm{CH}_{2}\right)$, 4.46 (s, 2H, $\mathrm{OCH}_{2} \mathrm{CO}$ ), 7.08 (br. s, 1H, H-7), 7.27 (br. d, $J=8.3 \mathrm{~Hz}, \mathrm{H}-5$ ), 7.44 (d, $J=8.3 \mathrm{~Hz}$, $\mathrm{H}-4), 8.05$ (br. t, $J=6.0 \mathrm{~Hz}, 1 \mathrm{H}, \mathrm{NH}) .{ }^{13} \mathrm{C} \mathrm{NMR}$ $\left(\mathrm{CDCl}_{3}\right): 25.2,34.2,35.0,37.1,67.6,106.8$, 124.0, 128.4, 138.3, 148.8, 157.8, 167.9, 173.3, 206.5. IR (KBr, v, $\left.\mathrm{cm}^{-1}\right): 3333,2924,1712$, 1650, 1564, 1488, 1427, 1284, 1191, 1063, 845.

General procedure for $\mathrm{N}$-acylation of the amino acid methyl esters by acid 6 through the activated imidazole derivatives. A solution of $0.21 \mathrm{~g}(1 \mathrm{mmol})$ of acid 6 and $0.2 \mathrm{~g}$ (1.25 mmol) 1,1'-carbonyldiimidazole in $10 \mathrm{ml}$ of absolute dioxane vigorous stirring at room temperature for $2-2.5 \mathrm{~h}$. To the resulting activated imidazole derivative $1.1 \mathrm{mmol}$ of the corresponding ester of amino acid (hydrochloride) and $0.17 \mathrm{ml}(1.25 \mathrm{mmol})$ of $\mathrm{Et}_{3} \mathrm{~N}$ were added. The reaction mixture was stirred at $50^{\circ} \mathrm{C}$ for $2-3 \mathrm{~h}$, then cooled and poured into $50 \mathrm{ml}$ of water and. The slowlyoxy]acetyl $\}$ glycine 10a. ${ }^{1} \mathrm{H} \mathrm{NMR}\left(\mathrm{CDCl}_{3}\right): 2.75$ (br. t, $J=5.6 \mathrm{~Hz}, 2 \mathrm{H}, \mathrm{H}_{2}-3$ ), 3.11 (br. t, $J=5.6 \mathrm{~Hz}$, $\left.2 \mathrm{H}, \mathrm{H}_{2}-2\right), 3.80(\mathrm{~s}, 3 \mathrm{H}, \mathrm{COOMe}), 4.17$ (d, $\left.J=5.8 \mathrm{~Hz}, 2 \mathrm{H}, \mathrm{NHCH}_{2} \mathrm{COOH}\right), 4.59$ (s, 2H, $\left.\mathrm{OCH}_{2} \mathrm{CO}\right), 7.08(\mathrm{~m}, 1 \mathrm{H}, \mathrm{NH}), 7.24$ (d, $J=2.7 \mathrm{~Hz}, \quad 1 \mathrm{H}, \quad \mathrm{H}-7), \quad 7.27$ (dd, ${ }^{3} J=8.3 \mathrm{~Hz}$, $\left.{ }^{4} J=2.7 \mathrm{~Hz}, \mathrm{H}-5\right), 7.45$ (d, $\left.J=8.3 \mathrm{~Hz}, 1 \mathrm{H}, \mathrm{H}-4\right)$. ${ }^{13} \mathrm{C} \mathrm{NMR}\left(\mathrm{CDCl}_{3}\right): 25.2,37.0,40.8,52.5,67.5$, 107.0, 123.6, 127.9, 138.5, 149.2, 156.9, 168.0, 169.9, 206.5. IR (KBr, v, $\left.\mathrm{cm}^{-1}\right): 3546,3451$, 3322, 3036, 2913, 1731, 1706, 1662, 1552, 1490, 1446, 1245, 1071, 984, 834.

Methyl ester of $N-\{[(1-o x o i n d a n-6-y l)-$ oxy] acetyl $\}$ methionine 10c. ${ }^{1} \mathrm{H} \mathrm{NMR}\left(\mathrm{CDCl}_{3}\right)$ : 2.01-2.13 (m, 4H, $\left.\mathrm{NHCH}\left[\mathrm{CH}_{2} \underline{\mathrm{CH}}_{\alpha} \underline{\mathrm{SCH}}_{3}\right]\right)$, 2.19-2.27 (m, 1H, NHCH[C $\left.\left.\mathrm{CH}_{2} \underline{\mathrm{CH}_{\beta}} \mathrm{SCH}_{3}\right]\right), 2.52$ (t, J=7.3 Hz, $\left.2 \mathrm{H}, \mathrm{NHCH}\left[\underline{\mathrm{CH}}_{2} \mathrm{CH}_{2} \mathrm{SCH}_{3}\right]\right), 2.75$ (br. t, $J=5.6 \mathrm{~Hz}, 2 \mathrm{H}, \mathrm{H}_{2}-3$ ), 3.11 (br. t, $J=5.6 \mathrm{~Hz}$, $\left.2 \mathrm{H}, \mathrm{H}_{2}-2\right), 3.79$ (s, 3H, COOMe), 4.53-4.61 (m, $\left.2 \mathrm{H}, \quad \mathrm{OCH}_{2} \mathrm{CO}\right), \quad 4.80-4.86 \quad(\mathrm{~m}, \quad 1 \mathrm{H}$, $\left.\mathrm{NH} \underline{\mathrm{CH}}\left[\left(\mathrm{CH}_{2}\right)_{2} \mathrm{SCH}_{3}\right]\right), 7.24(\mathrm{~d}, J=2.0 \mathrm{~Hz}, 1 \mathrm{H}$, $\mathrm{H}-7), 7.25-7.30\left(2 \mathrm{H}+\mathrm{CHCl}_{3}, \mathrm{~m}, \mathrm{H}-5, \mathrm{NH}\right), 7.45$ (d, $J=8.5 \mathrm{~Hz}, 1 \mathrm{H}, \mathrm{H}-4) .{ }^{1} \mathrm{H}$ NMR (DMSO-d 6 ): 1.85-2.06 (m, 5H, $\left.\mathrm{NHCH}\left[\mathrm{CH}_{2} \underline{\mathrm{CH}}_{2} \mathrm{SCH}_{3}\right]\right)$, 2.34-2.50 (m, $\left.2 \mathrm{H}, \mathrm{NHCH}\left[\underline{\mathrm{CH}} 2 \mathrm{CH}_{2} \mathrm{SCH}_{3}\right]\right), 2.64$ (br. t, $J=5.6 \mathrm{~Hz}, 2 \mathrm{H}, \mathrm{H}_{2}-3$ ), 3.01 (br. t, $J=5.6 \mathrm{~Hz}$, $\left.2 \mathrm{H}, \mathrm{H}_{2}-2\right), 3.62$ (s, 3H, COOMe), 4.41-4.50 (m, $\left.1 \mathrm{H}, \mathrm{NHCH}\left[\left(\mathrm{CH}_{2}\right)_{2} \mathrm{SCH}_{3}\right]\right), 4.56-4.69(\mathrm{~m}, 2 \mathrm{H}$, $\mathrm{OCH}_{2} \mathrm{CO}$ ), 7.07 (br. s, 1H, H-7), 7.31 (br. d, $J=8.2 \mathrm{~Hz}, \mathrm{H}-5), 7.51$ (d, $J=8.2 \mathrm{~Hz}, \mathrm{H}-4), 8.59$ 
(d, $J=7.7 \mathrm{~Hz}, 1 \mathrm{H}, \mathrm{NH}) .{ }^{13} \mathrm{C}$ NMR (DMSO-d 6 ):

25.4, 30.5, 37.3, 51.2, 67.4, 106.7, 124.4, 128.5, $138.4,149.0,158.0,168.6,172.6,206.7$. IR

$\left(\mathrm{KBr}, \mathrm{v}, \mathrm{cm}^{-1}\right): 3417,3064,2952,2913,1731$, $1712,1688,1614,1524,1488,1443,1362$, 1250, 1166, 1057, 990, 903, 831.

\section{General procedure for methylation of} COOH group of acids 8. To a solution of $0.75 \mathrm{mmol}$ of acid $8 \mathrm{in} 10 \mathrm{ml}$ of acetone $0.22 \mathrm{~g}$ $(1.6 \mathrm{mmol})$ of dried and powdered $\mathrm{K}_{2} \mathrm{CO}_{3}$ and $0.1 \mathrm{ml}(1.6 \mathrm{mmol})$ of MeI were added. The reaction mixture was refluxed with vigorous stirring $1.5-2 \mathrm{~h}$. After the process was finished (TLC monitoring) the solution of product was separated from inorganic precipitate, and evaporated in vacuum. The residue was recrystallized from mixture $i-\mathrm{PrOH}-$ water $1: 1$.

\section{Conclusions}

So it was shown that the $N$-acylation of amino acids by 2-(3-oxo-2,3-dihydro- $1 H$-inden5-yloxy)acetic acid can be easily made using the method of $N$-hydroxysuccinimide esters; and it performed the creation of new indan-1-one derivatives with various amino acid fragments possible. For synthesis of appropriate methyl esters not reaction between 2-(3-oxo-2,3dihydro- $1 H$-inden-5-yloxy)acetic acid activated imidazole derivative and amino acids methyl esters but the methylation of the carboxylic function of preformed $N$-\{[(1-oxoindan-6-yl)oxy] acetyl $\}$ amino acids can be used.

\section{References}

[1] Yoshishira K, Fukuoka M, Kuroyanagi M, Natori S. Chemical and Toxicological Studies on Bracken Fern, Pteridium aquilinum var. latiusculum. I. Introduction, Extraction and Fractionation of Constituents, and Toxicological Studies including Carcinogenicity Tests. Chem. Pharm. Bull. 1978; 26(8): 2346-2364.

[2] Padwa A, Curtis EA, Sandanayaka VP, Weingarten MD. A New Synthetic Route to the Illudin and Pterosin Family of Sesquiterpenes. Paper presented at: Electronic Conference Trends Org. Chem., 1995 Jun 12 Jul 7. The Royal Society of Chemistry: Cambridge, 1996. Keynote Paper 1. Available from: https://www.ch.ic.ac.uk/ectoc/papers/01/.

Also see: Canary JW. Electronic Conference on Trends in Organic Chemistry: ECTOC-1/CD-ROM. June 12-July 7, 1995 Edited by Henry S. Rzepa, Christopher Leach, and Jonathan M. Goodman. The Royal Society of Chemistry: Cambridge, 1996. J. Am. Chem. Soc. 1997; 119(19): 4567.

[3] Allegrini P, Mantegazza S, Razzetti G, inventor; Dipharma Francis S.r.l., assignee. A process for the preparation of delapril. European patent 1878740 . 2008 Jan 16.

[4] Birch AM, Bennett SNL, Campbell AD, Simpson I, Whittamore PRO, Whalley DP, Godfrey L, inventor; Astrazeneca $\mathrm{AB}$, Astrazeneca UK Limited, assignee. Heterocyclic amide derivatives which possess glycogen phosphorylase inhibitory activity. WO2005/013981. 2005 Feb 17.

[5] Tam, TF, Leung-Toung R, Wang Y, Zhao Y, Xin T, Li W, Wodzinska JM, Rabadia VS, Feeney CJ, inventor; Apotex Technologies Inc., assignee. Prodrugs of D-isoglutamyl-[D/L]-tryptophan. WO2012/129671. 2012 Oct 4.

[6] Ingold CK, Piggott HA. CLXX.-The mobility of symmetrical triad systems. Part II. The conditions relating to systems terminated by the $o$ - 
phenylene group. Derivatives of indene. J. Chem. Soc. 1923; 123: 1469-1509.

[7] Turek M, Szczęsna D, Koprowski M, Bałczewski P. Synthesis of 1-indanones with a broad range of biological activity. Beilstein J. Org. Chem. 2017; 13: 451-494.

[8] Weinstock M, Bejar C, Wang RH, Poltyrev T, Gross A, Finberg JP, Youdim MB. TV3326, a novel neuroprotective drug with cholinesterase and monoamine oxidase inhibitory activities for the treatment of Alzheimer's disease. J. Neural. Transm. Suppl. 2000; (60): 157-69.

[9] Zhu L-J, Hou Y-L, Shen X-Y, Pan X-D, Zhang X, Yao X-S. Monoterpene pyridine alkaloids and phenolics from Scrophularia ningpoensis and their cardioprotective effect. Fitoterapia 2013; 8: 44-49.

[10] Koelsch FC, Scheiderbauer RA. The Tautomerism of Indene. J. Am. Chem. Soc. 1943; 65(12): 2311-2314.

[11] Bjrnstad V, Undheim K. Cyclic Ketones in Spiroannulation. Synth. Comm. 2009; 39(10): 17931800.

[12] Anderson GW, Zimmerman JE, Callahan FM. N-Hydroxysuccinimide Esters in Peptide Synthesis. J. Am. Chem. Soc. 1963; 85(19): 3039.

[13] Gershkovich AA，Kibirev VK. Chemical Synthesis of Peptides [in Russian]. Kyiv: Naukova Dumka; 1992, pp. 38-44. 